\title{
Electrostatic self-energy and Bekenstein entropy bound in the massive Schwinger model
}

\author{
H. Mohseni Sadjadi*and M.Alimohammadi ${ }^{\dagger}$ \\ Department of Physics, University of Tehran, \\ North Karegar Ave., Tehran, Iran.
}

September 17, 2018

\begin{abstract}
We obtain the electrostatic energy of two opposite charges near the horizon of stationary black-holes in the massive Schwinger model. Besides the confining aspects of the model, we discuss the Bekenstein entropy upper bound of a charged object using the generalized second law. We show that despite the massless case, in the massive Schwinger model the entropy of the black hole and consequently the Bekenstein bound are altered by the vacuum polarization.
\end{abstract}

\section{Introduction}

Using the generalized second law for black holes, a universal upper bound on the entropy of a macroscopic charged object can be obtained by a gedanken experiment known as Geroch process. In this process the object is lowered adiabatically toward the horizon and then is assimilated from a small proper distance into the hole 1],2]. On the basis of the no-hair theorem it is claimed that this bound cannot be improved by other quantum numbers which are carried by the object, e.g. the baryon number [3]. A direct proof for the special case of a particle with a scalar charge, is given in [4. This proof is based on the fact that the self-energy receives no contribution from the scalar charge and the entropy of the assimilated object consists only of its gravitational energy.

A quantum effect relevant to this subject is the radiative correction involving the loop graphs, the so called vacuum polarization. Because of computational difficulties in studying the quantum effects in four dimensional curved spacetimes, one can consider lower dimensional models as a framework to explore these effects and to obtain the physical clues to the real four dimensional cases.

In [5] it is shown that in the massless Schwinger model [6], the Bekenstein entropy upper bound is not affected by vacuum polarization. This lies on the fact that the effect of vacuum polarization is only appeared in the mass gained by the gauge field. This can not change the entropy upper bound since the gauge field behaves like a conformal massless field near the horizon.

\footnotetext{
*hmohseni@phymail.ut.ac.ir

†alimohmd@ut.ac.ir
} 
In this paper, using the generalized second law for black-holes, we compute the entropy upper bound of a charged macroscopic object in the massive Schwinger model [7-12].

The paper is organized as follows: The section two, is devoted to a brief introduction of massive Schwinger model in curved space-time. In the section three we discuss the confining and screening aspects of this model in static black-hole background. We show that our results asymptotically reduce to ones obtained in 13] where the WKB approximation has been used. Also we compute the electrostatic self-energy of a charged particle very close to the horizon in terms of Bessel functions. These results are used to check the more complicated expression of self-energy derived in the section four where a term proportional to scalar curvature is included in the metric expansion. Our method is based on Taylor expansion of the metric in Schwarzschild gauge near the horizon, leading to Rindler (section three) or AdS space-time (section four) depending on the order of our approximation. Finally in the section five, we find out when an external charge is assimilated into the hole, the massive dynamical fermions affects the hole's entropy via the self-energy of the charge. This effect disappears when dynamical fermions are massless.

In this paper the charges of the hole and the object are assumed to be very small in the scale of the hole mass. The units $c=G=\hbar=1$ are used throughout the paper.

\section{2 preliminaries}

Since all two dimensional spaces are conformally flat, the most general stationary metric reads

$$
d s^{2}=\sqrt{g(x)}\left(d t^{2}-d x^{2}\right)
$$

where $g(x)=\left|\operatorname{det} g_{\mu \nu}(x)\right|$, and $g_{\mu \nu}$ depends only on the spatial coordinate $x$. On this space-time, the quantum electrodynamics of fermions of mass $m$ and charge $e$ is described by the action [10, 13]

$$
S=\int\left[-\frac{1}{4} g^{\mu \nu} g^{\lambda \beta} F_{\mu \lambda} F_{\nu \beta}+i \bar{\psi} \gamma^{\mu}\left(\nabla_{\mu}-i e A_{\mu}\right) \psi-m \bar{\psi} \psi\right] \sqrt{g(x)} d x d t .
$$

$\gamma^{\mu}=e^{\mu a} \gamma_{a}$ is the curved space-time counterparts of Dirac gamma matrices $\gamma_{a}$ and the zweibeins $e^{\mu a}$ 's satisfy $e^{\mu a} e_{\nu a}=\delta_{\nu}^{\mu}$. The covariant derivative, acting on fermions, is given by $\nabla_{\mu}=\partial_{\mu}+\frac{1}{2} \omega_{\mu}^{a b} \sigma_{a b}$ in which $\sigma_{a b}=\frac{1}{4}\left[\gamma_{a}, \gamma_{b}\right]$ and $\omega_{\mu}^{a b}$ 's are spin-connections. $F_{\mu \nu}=\partial_{\mu} A_{\nu}-\partial_{\nu} A_{\mu}$ are the field strength tensor components. The vacuum angle is assumed to be zero.

The corresponding bosonic action, in the presence of covariant conserved current

$$
J^{\mu}=\frac{e^{\prime}}{\sqrt{g(x)}}[\delta(x-a)-\delta(x-b)] \delta_{0}^{\mu}
$$

describing two opposite external charges $e^{\prime}$ and $-e^{\prime}$ located at $x=a$ and $x=b$, respectively, is 13 .

$$
\begin{aligned}
S= & \int\left[\frac{1}{2} \sqrt{g} g^{\mu \nu} \partial_{\mu} \phi \partial_{\nu} \phi+\frac{e}{\sqrt{\pi}} F \phi+\frac{m}{\pi} g^{\frac{1}{4}}(x) \exp [-2 \pi G(\mathbf{x}, \mathbf{x})]\right. \\
& \left.N_{\mu} \cos (2 \sqrt{\pi} \phi)+\frac{1}{2 \sqrt{g(x)}} F^{2}+\frac{e}{\sqrt{\pi}} \eta F\right] d x d t .
\end{aligned}
$$


The dual field strength $F$ is defined through $F=\hat{\epsilon}^{\mu \nu} \partial_{\mu} A_{\nu}$, where $\hat{\epsilon}^{01}=-\hat{\epsilon}^{10}=$ 1. $N_{\mu}$ is normal ordering with respect to scale

$$
\mu=\frac{e}{\sqrt{\pi}}
$$

and $G(\mathbf{x}, \mathbf{x}):=G_{\mu}(\mathbf{x}, \mathbf{x})-D(\mathbf{x}, \mathbf{x}), \mathbf{x}=(t, x) . G_{\mu}(\mathbf{x}, \mathbf{x})$ is the Green function of a massive scalar field of mass $\mu$ and

$$
D\left(\mathbf{x}_{i}, \mathbf{x}_{j}\right)=-\frac{1}{4 \pi} \ln \left|\mathbf{x}_{i}-\mathbf{x}_{j}\right|^{2} .
$$

In terms of step functions, $\eta$ is defined as

$$
\eta=\frac{\sqrt{\pi} e^{\prime}}{e}[\theta(x-b)-\theta(x-a)]
$$

In the Schwarzschild gauge, the metric

$$
d s^{2}=f(r) d t^{2}-\frac{1}{f(r)} d r^{2}, \quad r>h
$$

describes a black hole whose the horizon is located at $r=h . h$ is defined through $f(h)=0$. We assume that $f(r)$ is a positive $\mathcal{C}^{\infty}$ function for $r>h$. In tortoise coordinates $(t, x)$, defined by $d x^{2}=d r^{2} / f^{2}(r)$, the metric (6) reduces to (10) for $f(r)=\sqrt{g(x)}$. By integrating over the gauge fields of action (4), we obtain

$$
S_{\text {eff. }}=\int\left[\frac{1}{2} g^{\mu \nu} \partial_{\mu} \phi \partial_{\nu} \phi+m \Sigma N_{\mu} \cos (2 \sqrt{\pi} \phi)-\frac{\mu^{2}}{2}(\phi+\eta)^{2}\right] d r d t,
$$

where $g_{t t}=-1 / g_{r r}=f(r)$ and $\Sigma:=\exp [-2 \pi G(\mathbf{x}, \mathbf{x})] /\left(\pi f^{\frac{1}{2}}(r)\right)$ is the chiral condensate $\langle\bar{\psi} \psi>13$.

The electrostatic energy of external charges can be calculated by either performing a typical Wilson loop calculation, or by computing the ground state expectation value of the Hamiltonian in the presence of external source

$$
E_{\text {elec. }}:=<\Omega_{Q}\left|H_{Q}\right| \Omega_{Q}>-<\Omega_{0}\left|H_{0}\right| \Omega_{0}>.
$$

$H_{0}\left(H_{Q}\right)$ and $\mid \Omega_{0}>\left(\mid \Omega_{Q}>\right)$ are the Hamiltonian and the ground state in the absence (presence) of the test charges, respectively [14. In the static case, the energy of the system measured by an observer whose two-velocity is parallel to the global time-like Killing vector of the space-time, i.e. $u=\left(f^{-\frac{1}{2}}, 0\right)$, is

$$
E=\int T_{0}^{0} d r=-\int L d r .
$$

$T_{\mu}^{\nu}$ is the energy-momentum tensor and $L$ is the Lagrangian density. For small $\phi$, where $\cos (2 \sqrt{\pi} \phi) \simeq 1-2 \pi \phi^{2}$, the action (41) becomes quadratic with respect to the gauge fields (this can be seen by integrating out the fermionic degrees of freedom in (2) or the bosonic ones in (41)). Hence in the static case, the electrostatic energy of charges in terms of the gauge field Green function $\mathcal{G}\left(r, r^{\prime}\right)$ becomes [12]

$$
E_{\text {elec. }}=-\frac{e^{\prime 2}}{2}\left[\mathcal{G}\left(r_{a}, r_{a}\right)+\mathcal{G}\left(r_{b}, r_{b}\right)-2 \mathcal{G}\left(r_{a}, r_{b}\right)\right]
$$


$e^{\prime 2} \mathcal{G}\left(r_{a}, r_{b}\right)$ is the interaction energy, $E_{\text {int. }}$, of two opposite charges $e^{\prime}$ and $-e^{\prime}$ located at $r_{a}:=r(a)$ and $r_{b}:=r(b)$, and $-\left(e^{\prime 2} / 2\right) \mathcal{G}(r, r)$ is the self-energy $E_{\text {self. }}$. In this paper, by self-energy of a charge we mean the change of energy of the system when the charge is added to it. Note that in contrast to higher dimensional cases, $\mathcal{G}(x, x)$, where $x$ is the spatial coordinate of the two dimensional space-time, is not infinite. For example in the massless Schwinger model on flat space-time, we have $\mathcal{G}(x, y)=-1 /(2 \mu) \exp [-\mu|x-y|]$, which becomes the constant $\mathcal{G}(x, x)=-1 /(2 \mu)$ in the coincident limit [15].

Up to the first order of $m$ and when $m \Sigma \ll \mu^{2}$, the electrostatic energy of widely separated charges, i.e. $\eta=\left(e^{\prime} / e\right) \sqrt{\pi}$, is [7], 13]

$$
E_{\text {elec. }}=E(\eta)-E(0)=m\left[1-\cos \left(2 \pi \frac{e^{\prime}}{e}\right)\right] \int_{r_{a}}^{r_{b}} \Sigma d r .
$$

Note that the action is not quadratic here and the equation (10) is not valid. For constant values of $\eta$, using (44), one can show that the gauge fields with zero winding number $\left(\int F d r d t=0\right)$ do not contribute to the electrostatic energy of external charges.

For finitely separated charges, $\eta$ is no more a constant and the solution of the classical equation of motion for the field $|\phi| \ll 1$ is

$$
\phi=-\frac{\mu^{2}}{\partial_{r} g^{r r} \partial_{r}+\mu^{2}+4 \pi m \Sigma} \eta .
$$

In this regime, the action becomes Gaussian and the classical solutions of the action coincide with the quantum ones. Putting (12) back into Lagrangian (7), eq.(9) results

$$
E_{\text {elec. }}=\frac{\mu^{2}}{2}\left[\int_{r_{a}}^{r_{b}}\left(\eta^{2}-\mu^{2} \eta \frac{1}{\partial_{r} g^{r r} \partial_{r}+\mu^{2}+4 \pi m \Sigma} \eta\right) d r\right] .
$$

This equation can be rewritten as

$$
E_{\text {elec. }}=\left(\frac{e^{\prime}}{\mu}\right)^{2}\left[\frac{\mu^{2}}{2}\left(r_{b}-r_{a}\right)+\Pi\right]
$$

where

$$
\begin{aligned}
\Pi:=-\frac{\mu^{4}}{2} \int_{r_{a}}^{r_{b}} d r\left[\int_{r^{\prime}=r_{a}}^{r^{\prime}=r} G\left(r_{>}=r, r_{<}=r^{\prime}\right) d r^{\prime}\right. \\
\left.\quad+\int_{r^{\prime}=r}^{r^{\prime}=r_{b}} G\left(r_{>}=r^{\prime}, r_{<}=r\right) d r^{\prime}\right],
\end{aligned}
$$

and

$$
\left(\partial_{r} g^{r r} \partial_{r}+\mu^{2}+4 \pi m \Sigma\right) G\left(r, r^{\prime}\right)=\delta\left(r, r^{\prime}\right) .
$$

We denote the smaller (bigger) argument of the Green function by $>(<)$.

In [13, for a slowly varying metric $d f^{\frac{1}{2}}(r) / d r \ll \bar{\mu}$, where

$$
\bar{\mu}^{2}=\mu^{2}+4 \pi m \Sigma,
$$


$E_{\text {elec. }}$ has been derived as

$$
\begin{aligned}
E_{\text {elec. }} & =\frac{e^{\prime 2}}{2}\left(1-\frac{\mu^{2}}{\bar{\mu}^{2}}\right)\left(r_{b}-r_{a}\right)+\frac{e^{\prime 2} \mu^{2}}{4 \bar{\mu}^{3}}\left[f^{\frac{1}{2}}\left(r_{a}\right)\right. \\
& \left.+f^{\frac{1}{2}}\left(r_{b}\right)-2 f^{\frac{1}{4}}\left(r_{a}\right) f^{\frac{1}{4}}\left(r_{b}\right) \exp \left[-\int_{r_{a}}^{r_{b}} \bar{\mu} f^{-\frac{1}{2}}(u) d u\right]\right] .
\end{aligned}
$$

The above equation has been obtained in the zeroth order of WKB approximation. In this small curvature limit, (18) is the leading term of the energy. If the metric were a constant, the self-force (the derivative of the self-energy) would vanish and the energy should become the same as the flat case, shifted by the metric factor $f^{1 / 2}$. This result is consistent with the measurement of a constantly accelerated observer. The condition of validity of (18) is $e^{\prime} \ll e$ and the first expression in (18) reproduces (11) in this limit.

Near the horizon, WKB approximation fails [16. This can be related to zero frequency modes of massive scalar fields appeared in the Schwinger model 5]. So, to obtain the energy of external charges near the horizon, we must use another approximation. Note that the solutions of (16) in regions near and far from the horizon must be matched asymptotically.

\section{$3 q \bar{q}$ potential near horizon in the massive Schwinger model}

Near the horizon of a non-extremal black-hole, we can expand the metric as

$$
f(r)=\kappa(r-h)+O(r-h)^{2},
$$

where $\kappa:=d f / d r$ is twice of the surface gravity. This metric describes Rindler space-time. In this region, $G\left(r, r^{\prime}\right)$ satisfies

$$
\left[-\partial_{r} \kappa(r-h) \partial_{r}+\mu^{2}+4 \pi m \Sigma\right] G\left(r, r^{\prime}\right)=\delta\left(r, r^{\prime}\right)
$$

where $\Sigma=\mu \exp (\gamma) /(2 \pi)[13$ and $\gamma$ is the Euler constant. Two independent solutions of the corresponding homogeneous equation are $I_{0}(2 \bar{\mu} \sqrt{(r-h) / \kappa})$

and $K_{0}(2 \bar{\mu} \sqrt{(r-h) / \kappa})$, where $I_{0}$ and $K_{0}$ are modified Bessel functions. The well-defined Green function is then

$$
G\left(r, r^{\prime}\right)=\frac{2}{\kappa} K_{0}\left(2 \bar{\mu} \sqrt{\frac{r_{>}-h}{\kappa}}\right) I_{0}\left(2 \bar{\mu} \sqrt{\frac{r_{<}-h}{\kappa}}\right) .
$$

Defining $\mathcal{K}$ and $\mathcal{I}$ through

$$
\begin{aligned}
\frac{d \mathcal{K}}{d r} & =K_{0}\left(2 \bar{\mu} \sqrt{\frac{r-h}{\kappa}}\right), \\
\frac{d \mathcal{I}}{d r} & =I_{0}\left(2 \bar{\mu} \sqrt{\frac{r-h}{\kappa}}\right)
\end{aligned}
$$


then $\Pi$ in (15) reduces to

$$
\begin{aligned}
\Pi & =-\frac{\mu^{4}}{\kappa}\left\{\int_{r_{a}}^{r_{b}} W[\mathcal{I}(r), \mathcal{K}(r)] d r\right. \\
& \left.+\left[\mathcal{I}\left(r_{b}\right) \mathcal{K}\left(r_{b}\right)+\mathcal{I}\left(r_{a}\right) \mathcal{K}\left(r_{a}\right)-2 \mathcal{I}\left(r_{a}\right) \mathcal{K}\left(r_{b}\right)\right]\right\},
\end{aligned}
$$

where $W$ is the Wronskian. Considering the recurrence formulas

$$
\frac{d}{d x}\left[x^{n} I_{n}(x)\right]=x^{n} I_{n-1}(x), \quad \frac{d}{d x}\left[x^{n} K_{n}(x)\right]=-x^{n} K_{n-1}(x),
$$

one can show

$$
\begin{aligned}
& \mathcal{K}(r)=-\frac{\sqrt{\kappa}}{\bar{\mu}}(r-h)^{\frac{1}{2}} K_{1}\left(2 \bar{\mu} \sqrt{\frac{r-h}{\kappa}}\right), \\
& \mathcal{I}(r)=\frac{\sqrt{\kappa}}{\bar{\mu}}(r-h)^{\frac{1}{2}} I_{1}\left(2 \bar{\mu} \sqrt{\frac{r-h}{\kappa}}\right) .
\end{aligned}
$$

Using $W\left[K_{1}(x), I_{1}(x)\right]=1 / x$, we arrive at $W[\mathcal{I}(r), \mathcal{K}(r)]=\kappa /\left(2 \bar{\mu}^{2}\right)$. Therefore

$$
\begin{aligned}
E_{\text {elec. }} & =\frac{e^{\prime 2}}{2}\left(1-\left(\frac{\mu}{\bar{\mu}}\right)^{2}\right)\left(r_{b}-r_{a}\right) \\
& -\frac{e^{\prime 2} \mu^{2}}{\kappa}\left[\mathcal{I}\left(r_{b}\right) \mathcal{K}\left(r_{b}\right)+\mathcal{I}\left(r_{a}\right) \mathcal{K}\left(r_{a}\right)-2 \mathcal{I}\left(r_{a}\right) \mathcal{K}\left(r_{b}\right)\right] .
\end{aligned}
$$

The first and the last terms describe the interaction of opposite charges. While the first term corresponds to confinement aspects of the model, the last term illustrates the screening effect. Note that both the screening and confining phenomenon appear in the same problem, similar to what happened in the flat case [17. For $m=0(\mu=\bar{\mu})$, the confining term disappears. The second and the third terms are the self-energies of the charges. Writing eq.(25) in the form (10), we get

$$
\mathcal{G}\left(r, r^{\prime}\right)=\frac{1}{2}\left[1-\left(\frac{\mu}{\bar{\mu}}\right)^{2}\right]\left(r_{>}-r_{<}\right)+\frac{2 \mu^{2}}{\kappa} \mathcal{I}\left(r_{<}\right) \mathcal{K}\left(r_{>}\right) .
$$

$\left(2 \mu^{2} / \kappa\right) \mathcal{I}\left(r_{<}\right) \mathcal{K}\left(r_{>}\right)$is the only term which contributes to the self-energy in (25). As this part of the Green function satisfies Dirichlet boundary condition at the horizon, we find that $E_{\text {self. }}(r \rightarrow h)=0$. Far from the horizon, WKB approximation is applicable and from (18) we obtain $E_{\text {self. }}(r \gg h)=e^{\prime 2} \mu^{2} /\left(4 \bar{\mu}^{3}\right)$.

We can use the global method of Smith and Will 18 to determine the selfforce. In a free falling system, the work done by the force $F$ to displace slowly (such that the location of the event horizon remains unchanged) the test charge by an infinitesimal distance $\delta \bar{r}$ toward the horizon is

$$
\delta \bar{W}=-F \delta \bar{r} .
$$

The corresponding energy detected by an observer at asymptotic infinity will be red-shifted

$$
\delta E=\sqrt{g_{t t}(r)} \delta \bar{W}
$$


This change will be manifested by a change in the asymptotic mass $\mathbf{M}$ of the system, given by the total mass variation law of Carter 19.

$$
\delta \mathbf{M}=\delta \int_{h}^{\infty} T_{t}^{t} d r,
$$

where $T_{t}^{t}$ is the component of energy momentum tensor and is the same as the effective Lagrangian in (7). Therefore $\delta \mathbf{M}=\delta E_{\text {self. }}$. Hence by transforming locally the flat coordinates denoted by $\bar{r}$ to the Schwarzschild ones, we obtain

$$
F=\frac{\delta E_{\text {self. }}}{\delta r} .
$$

To derive (25) we have assumed $|\phi(r)| \ll 1$. Let us check this assumption more carefully. Note that $\phi$ is the solution of the equation (12), obtained using eqs.(21) and (31) as:

$$
\phi(r)= \begin{cases}-\frac{2 \mu e^{\prime}}{\kappa} I_{0}\left(2 \bar{\mu} \sqrt{\frac{r-h}{\kappa}}\right)\left[\mathcal{K}\left(r_{b}\right)-\mathcal{K}\left(r_{a}\right)\right] & r<r_{a}<r_{b}, \\ -\frac{2 \mu e^{\prime}}{\kappa} K_{0}\left(2 \bar{\mu} \sqrt{\frac{r-h}{\kappa}}\right)\left[\mathcal{I}\left(r_{b}\right)-\mathcal{I}\left(r_{a}\right)\right] & \\ -\frac{2 \mu e^{\prime}}{\kappa}\left[K_{0}\left(2 \bar{\mu} \sqrt{\frac{r-h}{\kappa}}\right) \mathcal{I}(r)-I_{0}\left(2 \bar{\mu} \sqrt{\frac{r-h}{\kappa}}\right) \mathcal{K}(r)+\right. & \\ \left.+I_{0}\left(2 \bar{\mu} \sqrt{\frac{r-h}{\kappa}}\right) \mathcal{K}\left(r_{b}\right)-K_{0}\left(2 \bar{\mu} \sqrt{\frac{r-h}{\kappa}}\right) \mathcal{I}\left(r_{a}\right)\right] & r_{a}<r<r_{b} .\end{cases}
$$

The expressions appeared in $\phi(r)$ are in the forms

$$
\mathcal{F}_{1}=\frac{2 \mu e^{\prime}}{\kappa} K_{0}\left(2 \bar{\mu} \sqrt{\frac{r_{>}-h}{\kappa}}\right) \mathcal{I}\left(r_{<}\right),
$$

and

$$
\mathcal{F}_{2}=\frac{2 \mu e^{\prime}}{\kappa} I_{0}\left(2 \bar{\mu} \sqrt{\frac{r_{<}-h}{\kappa}}\right) \mathcal{K}\left(r_{>}\right) .
$$

Using the following expansions near the horizon [20,

$$
\begin{aligned}
K_{0}(x) & \sim-\left[\gamma+\ln \left(\frac{x}{2}\right)\right]\left(1+\frac{x^{2}}{4}\right)+\frac{x^{2}}{4}+O\left(x^{4}\right), \\
K_{1}(x) & \sim \frac{1}{x}+\frac{x}{2}\left[\ln \left(\frac{x}{2}\right)-\frac{\psi(1)+\psi(2)}{2}\right]+O\left(x^{3}\right), \\
I_{0}(x) & \sim 1+\frac{x^{2}}{4}+O\left(x^{4}\right), \\
I_{1}(x) & \sim \frac{x}{2}+O\left(x^{3}\right)
\end{aligned}
$$


where $\psi$ is digamma function, one finds

$$
\begin{aligned}
\mathcal{F}_{1} & \sim-\frac{2 \mu e^{\prime}\left(r_{<}-h\right)}{\kappa}\left[\gamma+\ln \left(\bar{\mu} \sqrt{\frac{r_{>}-h}{\kappa}}\right)\right], \\
\mathcal{F}_{2} & \sim \mu e^{\prime}\left\{\frac{1}{\bar{\mu}^{2}}+\frac{r_{<}-h}{\kappa}\right. \\
+ & \left.\frac{2\left(r_{>}-h\right)}{\kappa}\left[\ln \left(\bar{\mu} \sqrt{\frac{r_{>}-h}{\kappa}}\right)-\frac{\psi(1)+\psi(2)}{2}\right]\right\} .
\end{aligned}
$$

This shows that the assumption $|\phi| \ll 1$ is applicable near the horizon, in which $r \rightarrow h$.

From the asymptotic expansions [20]

$$
\begin{aligned}
I_{\nu}(z) & \sim \frac{1}{\sqrt{2 \pi}} z^{\nu}\left(-z^{2}\right)^{-\frac{1}{4}(2 \nu+1)}\left[e^{-i\left[\sqrt{-z^{2}}-\frac{1}{4}(2 \nu+1) \pi\right]}\left(1+O\left(z^{-1}\right)\right)\right. \\
+ & \text { c.c. }] \\
K_{\nu}(z) \quad & \sim \sqrt{\frac{\pi}{2 z}} e^{-z}\left[1+O\left(z^{-1}\right)\right],
\end{aligned}
$$

one can show that the eq.(25) asymptotically reduces to (18), as expected. This is the requirement explained at the end of section 2 .

\section{Electrostatic self-energy in the second order approximation}

To study the effect of the curvature in higher order approximation of self-energy, we must improve and refine the approximation (19) by including terms proportional to the scalar curvature in the metric expansion near the horizon $r \simeq h$. We put

$$
f(r)=\kappa(r-h)+\frac{R}{2}(r-h)^{2}+O\left((r-h)^{3}\right),
$$

where $\kappa=f^{\prime}(h)>0$ and $R=f^{\prime \prime}(h)$. This describes a space-time with constant curvature $-R$ (locally AdS or dS, depending on whether the sign of $-R$ is negative or positive, respectively). In terms of the coordinate $u=x+b$, where

$$
b=\frac{\kappa}{R}, \quad x=r-h,
$$

the equation (16) becomes

$$
\left[-\partial_{u} \frac{R}{2}\left(u^{2}-b^{2}\right) \partial_{u}+\bar{\mu}^{2}\right] G\left(u, u^{\prime}\right)=\delta\left(u, u^{\prime}\right) .
$$

In space-times with a constant positive curvature, $\Sigma$ is a constant [21]:

$$
\Sigma=\frac{\mu e^{\gamma}}{2 \pi} \exp \left[\frac{1}{2}\left\{\ln \left(-\frac{R}{2 \mu^{2}}\right)+\psi\left(\frac{1}{2}+\alpha\right)+\psi\left(\frac{1}{2}-\alpha\right)\right\}\right],
$$


where $\alpha^{2}=1 / 4+2 \mu^{2} / R$. We restrict ourselves to small negative $R$, which we encounter in the next section where a dilatonic black hole with a large mass is considered. For $|R| \ll \mu^{2}$, the chiral condensate becomes

$$
\Sigma=\frac{e^{\gamma} \mu}{2 \pi} \exp \left[\frac{R}{12 \mu^{2}}\right] .
$$

Defining

$$
z:=\frac{u}{b}, 0<z<1 \quad ; \tilde{\mu}^{2}:=\frac{-2 \bar{\mu}^{2}}{R}
$$

the homogeneous counterpart of the equation (35) becomes

$$
\left[\left(1-z^{2}\right) \partial_{z}^{2}-2 z \partial_{z}-\tilde{\mu}^{2}\right] G_{h}(z)=0 .
$$

The real solutions of (38) can be expressed in terms of conical functions defined by 22 ]

$$
\begin{aligned}
p_{\tilde{\nu}}^{n}(z) & =P_{\tilde{\nu}}^{-n}(z), \\
q_{\tilde{\nu}}^{n}(z) & =\frac{(-1)^{n}}{2}\left[Q_{\tilde{\nu}}^{n}(z)+Q_{-\tilde{\nu}-1}^{n}(z)\right] \\
& =-\frac{\pi}{2 \sin (\pi \tilde{\nu})} P_{\tilde{\nu}}^{n}(-z) .
\end{aligned}
$$

$P_{\tilde{\nu}}^{n}(z)$ and $Q_{\tilde{\nu}}^{n}(z)$ are associated Legendre functions and $\tilde{\nu}$ is defined through $\tilde{\nu}(\tilde{\nu}+1)=-\tilde{\mu}^{2}$. Note that in the small curvature limit $-2 \bar{\mu}^{2} / R>\frac{1}{4}$ or $\tilde{\mu}^{2}>\frac{1}{4}$, $\tilde{\nu}$ is a complex number. Near the horizon, $z=1$, we have [20]

$$
\begin{aligned}
\lim _{z \rightarrow 1} q_{\tilde{\nu}}(z)= & -\frac{1}{2} \ln \left(\frac{1-z}{2}\right)[1+O(1-z)]- \\
& \frac{1}{2}[\psi(1+\tilde{\nu})+\psi(-\tilde{\nu})+2 \gamma][1+O(1-z)], \\
\lim _{z \rightarrow 1} p_{\tilde{\nu}}(z)= & 1-\frac{\tilde{\nu}(\tilde{\nu}+1)}{2}(1-z)+O(1-z)^{2} .
\end{aligned}
$$

Hence the real well behaved Green function is

$$
G\left(r, r^{\prime}\right)=\frac{2}{\kappa} p_{\tilde{\nu}}\left(z_{>}\right) q_{\tilde{\nu}}\left(z_{<}\right)
$$

To arrive at this relation, we have used $W\left[p_{\tilde{\nu}}^{n}(z), q_{\tilde{\nu}}^{n}(z)\right]=1 /\left(1-z^{2}\right)$ [22].

Let us compare this result with one obtained in the previous section. In terms of $\theta$ defined by $z=\cos \theta$, (41) becomes

$$
G\left(z, z^{\prime}\right)=\frac{2}{\kappa} p_{\tilde{\nu}}\left(\cos \theta_{<}\right) q_{\tilde{\nu}}\left(\cos \theta_{>}\right) .
$$

One can make use of the relations 22]

$$
\begin{aligned}
\lim _{\theta \rightarrow 0} \tilde{\nu}^{n} p_{\tilde{\nu}}^{n}(\cos \theta) & =i^{n} I_{n}(n \lambda), \\
\lim _{\theta \rightarrow 0} \tilde{\nu}^{-n} q_{\tilde{\nu}}^{n}(\cos \theta) & =i^{-n} K_{n}(n \lambda),
\end{aligned}
$$

where $n \lambda /(\sin \theta)=\tilde{\mu}$, to obtain

$$
G\left(z, z^{\prime}\right)=\frac{2}{\kappa} I_{0}\left(\tilde{\mu} \theta_{<}\right) K_{0}\left(\tilde{\mu} \theta_{>}\right),
$$


in the vicinity of the horizon, i.e. $\theta \rightarrow 0$. In this region we have also $x \approx-\theta^{2} b / 2$ which yields $\theta \tilde{\mu}=2 \sqrt{x \bar{\mu}^{2} / \kappa}$. Therefore in the limit $x \rightarrow 0$, (41) tends to (21).

Putting back (41) into (15) we get

$$
\Pi=-\frac{\mu^{4}}{\kappa} W[\mathcal{P}, \mathcal{Q}]\left(r_{b}-r_{a}\right)-\frac{\mu^{4}}{\kappa}\left[\mathcal{P}\left(r_{a}\right) \mathcal{Q}\left(r_{a}\right)+\mathcal{P}\left(r_{b}\right) \mathcal{Q}\left(r_{b}\right)-2 \mathcal{P}\left(r_{a}\right) \mathcal{Q}\left(r_{b}\right)\right],
$$

where

$$
\begin{aligned}
\frac{d \mathcal{Q}(r)}{d r} & =q_{\tilde{\nu}}\left(1+\frac{r-h}{b}\right), \\
\frac{d \mathcal{P}(r)}{d r} & =p_{\tilde{\nu}}\left(1+\frac{r-h}{b}\right) .
\end{aligned}
$$

With the help of the recurrence formulas [20, 23]

$$
\begin{aligned}
\left(1-z^{2}\right) \frac{d P_{\tilde{\nu}}^{-1}(z)}{d z} & =-\tilde{\nu} z P_{\tilde{\nu}}^{-1}(z)+(\tilde{\nu}-1) P_{\tilde{\nu}-1}^{-1}(z), \\
\left(1-z^{2}\right)^{\frac{1}{2}} P_{\tilde{\nu}}(z) & =z(\tilde{\nu}+1) P_{\tilde{\nu}}^{-1}(z)-(\tilde{\nu}-1) P_{\tilde{\nu}-1}^{-1}(z),
\end{aligned}
$$

and

$$
\begin{aligned}
\left(1-z^{2}\right) \frac{d q_{\tilde{\nu}}^{1}(z)}{d z} & =-\tilde{\nu} z q_{\tilde{\nu}}^{1}(z)+(\tilde{\nu}+1) q_{\tilde{\nu}-1}^{1}(z), \\
-\tilde{\nu}\left(1-z^{2}\right)^{\frac{1}{2}} q_{\tilde{\nu}}(z) & =q_{\tilde{\nu}-1}^{1}(z)-z q_{\tilde{\nu}}^{1}(z),
\end{aligned}
$$

one obtains

$$
\begin{aligned}
\mathcal{Q}(r) & =-\frac{b}{\tilde{\nu}(\tilde{\nu}+1)}\left(1-z^{2}\right)^{\frac{1}{2}} q_{\tilde{\nu}}^{1}(z), \\
\mathcal{P}(r) & =-b\left(1-z^{2}\right)^{\frac{1}{2}} p_{\tilde{\nu}}^{1}(z) .
\end{aligned}
$$

The first equation of (48) is derived directly from the first identity of (47). To verify the second equation, we write it in terms of associated Legendre function $P$ as

$$
-P_{\tilde{\nu}-1}^{1}(z)+z P_{\tilde{\nu}}^{1}(z)=-\tilde{\nu}\left(1-z^{2}\right)^{\frac{1}{2}} P_{\tilde{\nu}}(z) .
$$

Using [23]

$$
z P_{\tilde{\nu}}^{\mu}(z)-P_{\tilde{\nu}+1}^{\mu}(z)=(\mu+\tilde{\nu})\left(1-z^{2}\right)^{\frac{1}{2}} P_{\tilde{\nu}}^{\mu-1}(z),
$$

(50) reduces to

$$
-P_{\tilde{\nu}-1}^{1}(z)+z P_{\tilde{\nu}}^{1}(z)=-\frac{\tilde{\nu}}{\tilde{\nu}+1}\left[z P_{\tilde{\nu}}^{1}(z)-P_{\tilde{\nu}+1}^{1}(z)\right] .
$$

But (52) is nothing but the known equation: 20]

$$
P_{\nu}^{\mu}(z)=\frac{2 \nu+3}{\mu+\nu+1} z P_{\nu+1}^{\mu}(z)+\frac{\mu-\nu-2}{\nu+\mu+1} P_{\nu+2}^{\mu}(z),
$$

when one takes $\nu=\tilde{\nu}-1$ and $\mu=1$. 
Using $W[\mathcal{P}, \mathcal{Q}]=b /[\tilde{\nu}(\tilde{\nu}+1)], E_{\text {elec. }}$ from (14) and (45) becomes

$$
\begin{aligned}
E_{\text {elec. }} & =\frac{e^{\prime 2}}{2}\left(1-\left(\frac{\mu}{\bar{\mu}}\right)^{2}\right)\left(r_{b}-r_{a}\right) \\
& -\frac{e^{\prime 2} \mu^{2}}{\kappa}\left[\mathcal{P}\left(r_{b}\right) \mathcal{Q}\left(r_{b}\right)+\mathcal{P}\left(r_{a}\right) \mathcal{Q}\left(r_{a}\right)-2 \mathcal{P}\left(r_{a}\right) \mathcal{Q}\left(r_{b}\right)\right] .
\end{aligned}
$$

From $\Gamma(\tilde{\nu}) \Gamma(1-\tilde{\nu})=\pi /[\sin (\pi \tilde{\nu})]$, it follows that [20]

$$
\begin{aligned}
\lim _{z \rightarrow 1} p_{\tilde{\nu}}^{1}(z) & =(1+z)^{-\frac{1}{2}}\left[(1-z)^{\frac{1}{2}}-\frac{\tilde{\nu}(\tilde{\nu}+1)}{2 \Gamma(3)}(1-z)^{\frac{3}{2}}\right]+O(1-z)^{\frac{5}{2}}, \\
\lim _{z \rightarrow 1} q_{\tilde{\nu}}^{1}(z) & =-\frac{\tilde{\nu}(\tilde{\nu}+1)}{2}(1+z)^{-\frac{1}{2}}(1-z)^{\frac{1}{2}}\left[\ln \left(\frac{1-z}{2}\right)-\psi(2)+\gamma+\right. \\
\psi(-\tilde{\nu}) & +\psi(\tilde{\nu}+1)][1+O(1-z)]+(1+z)^{-\frac{1}{2}}(1-z)^{-\frac{1}{2}} .
\end{aligned}
$$

We have also [20]

$$
\begin{aligned}
\psi(-\tilde{\nu})+\psi(\tilde{\nu}+1) & =\psi\left(\frac{1}{2}+\frac{i \lambda}{2}\right)+\psi\left(\frac{1}{2}-\frac{i \lambda}{2}\right) \\
& =2 \Re\left(\psi\left(\frac{1}{2}+\frac{i \lambda}{2}\right)\right),
\end{aligned}
$$

where $\lambda=\sqrt{4 \tilde{\mu}^{2}-1}$. For slowly varying metrics $\lambda \gg 1$, one can use the expansion $\psi(w) \sim \ln w-(1 / 2 w)-1 /\left(12 w^{2}\right)+O\left(w^{-4}\right)$, 20], to arrive at

$$
\psi(-\tilde{\nu})+\psi(\tilde{\nu}+1)=\ln \frac{-2 \bar{\mu}^{2}}{R}+\frac{8 \bar{\mu}^{2} R-R^{2}}{48 \bar{\mu}^{4}}+O\left(\frac{R^{3}}{\bar{\mu}^{6}}\right) .
$$

Combining (54)-(57) one finds

$$
\begin{aligned}
E_{\text {self. }}(x) & =\frac{e^{\prime 2}}{2} \frac{\mu^{2}}{\bar{\mu}^{2}}\left\{x+\frac{\bar{\mu}^{2}}{\kappa} x^{2}\left[\ln \frac{\bar{\mu}^{2} x}{\kappa}+\gamma-\psi(2)+\frac{1}{2}+\frac{R}{6 \bar{\mu}^{2}}\right.\right. \\
& \left.\left.-\frac{R^{2}}{48 \bar{\mu}^{4}}+O\left(R^{3}\right)\right]\right\}+O\left(x^{3}\right) .
\end{aligned}
$$

Note that at $m=0$, where $\bar{\mu}=\mu$, the above expression reduces to one obtained in [5].

\section{$5 \quad$ Bekenstein entropy bound}

Our aim is now to use the relation (58) to obtain the upper entropy bound of a charged object. To do so, we allow the black hole to carry a charge $q$ and assume $q, e^{\prime}$ and $\mu$ to be very small with respect to the black hole mass. We

consider two dimensional dilatonic charged black hole with mass $M$ and charge $q$ :

$$
d s^{2}=\left(1-2 M e^{-r}+q^{2} e^{-2 r}\right) d t^{2}-\left(1-2 M e^{-r}+q^{2} e^{-2 r}\right)^{-1} d r^{2},
$$


This metric emerges in the heterotic string theory as a solution of the action

$$
S[g, \varphi, A]=\frac{1}{2} \int_{\mathcal{M}} d^{2} x \sqrt{g} e^{\varphi}\left(R+(\nabla \varphi)^{2}-\frac{1}{2} F_{\mu \nu} F^{\mu \nu}\right)-\int_{\partial \mathcal{M}} d x \sqrt{I} e^{\varphi} K,
$$

describing $2 d$ gravity coupled to dilatonic field $\varphi . K$ is the extrinsic curvature and $I$ is the induced metric on $\partial \mathcal{M}$, where $\mathcal{M}$ is the surface under study. The boundary term is added to make the variation procedure self-consistent. The metric (59) can support an electrostatic test charge [24. Thermodynamical quantities for this system can be obtained using the Massieu function, expressed in term of grand canonical partition function corresponding to the action [60) [25]. In $2 d$, the horizon surface of the black hole is a point and we cannot consider the area. Nevertheless it might be useful to think about the value of dilatonic field at the horizon, $\varphi_{h}$, as a quantity playing the rôle of the logarithm of an effective area. The event horizon of the black hole is located at

$$
h=\ln \left[M+\left(M^{2}-q^{2}\right)^{\frac{1}{2}}\right] .
$$

For $q>M$ we have a naked singularity. The entropy of the system is obtained as [25]

$$
S=2 \pi e^{\varphi_{h}}=2 \pi\left[M+\left(M^{2}-q^{2}\right)^{\frac{1}{2}}\right] .
$$

Comparing (59) with (6), gives $f(r)=1-2 M e^{-r}+q^{2} e^{-2 r}$. Expanding $f(r)$ near $r=h$, results $\kappa>0$ and $R<0$ for $q \ll M . \kappa$ and $R$ are defined through eq.(34).

We now consider a charged object of rest mass $m$ and charge $e$ whose gravitational field is negligible on this background. This object is slowly (adiabatically) descended toward the black hole. This process causes no change in the horizon location and the entropy of the black hole remains unchanged [3]. To find the change in black hole entropy caused by assimilation of the object, one should evaluate the energy at the point of capture, which is at a proper distance $l$ outside the horizon

$$
l=\int_{0}^{a} \frac{d y}{\left[\kappa y+\frac{R y^{2}}{2}\right]^{\frac{1}{2}}}=2 \sqrt{\frac{a}{\kappa}}-\frac{1}{6} R\left(\frac{a}{\kappa}\right)^{\frac{3}{2}}+O\left(\frac{a}{\kappa}\right)^{\frac{5}{2}} .
$$

$y$ is the coordinate distance from the horizon and $a$ is the position of the center of mass of the object. In fact $l$ is the proper radius of the object at the point of capture. For small $l$ we get $\sqrt{a / \kappa}=l / 2+(R / 96) l^{3}+O\left(l^{5}\right)$. When the object is assimilated, its charge modifies the hole's charge to $q+e^{\prime}$, and its total energy, which we denote by $E_{T}$, augments the hole's mass from $M$ to $M^{\prime}=M+E_{T}$.

The energy of the object, $E_{T}$, is constituted of the energy of the body's mass, $m^{\prime}$, shifted by the gravitational field, $E_{m^{\prime}} \simeq m^{\prime} \kappa l / 2+O\left(l^{3}\right)$, and the electrostatic self-energy $E_{\text {self. }}$ (58) which in terms of $l$ is:

$$
\begin{aligned}
E_{\text {self. }} & =\frac{e^{\prime 2}}{2} \frac{\mu^{2}}{\bar{\mu}^{2}}\left\{\frac{\kappa l^{2}}{4}+\frac{\kappa R l^{4}}{96}+\frac{\bar{\mu}^{2} \kappa l^{4}}{16}\left[2 \ln \left(\bar{\mu}\left(\frac{l}{2}+\frac{R l^{3}}{96}\right)\right)+\gamma-\psi(2)\right.\right. \\
& \left.\left.+\frac{1}{2}+\frac{R}{6 \bar{\mu}^{2}}-\frac{R^{2}}{48 \bar{\mu}^{4}}+O\left(R^{3}\right)\right]\right\}+O\left(l^{6}\right) .
\end{aligned}
$$


The final entropy of the black hole is

$$
S_{f}=2 \pi\left(2 M+2 E_{T}-\frac{q^{2}+e^{\prime 2}+2 q e^{\prime}}{2 M}+O\left(M^{-2}\right)\right) .
$$

In the massive Schwinger model, the fermionic parameters $\mu$ and $m$ are appeared in the final black hole entropy through the self-energy $E_{T}$. In contrast, in the massless Schwinger model, where $\bar{\mu}=\mu$, any information about fermionic field is lost (up to the order $l^{4}$ ). This result agrees with [5].

Assuming the validity of the generalized second law of thermodynamics [26]

$$
S_{f} \geq\left(S_{i}+S\right)
$$

where $S_{f(i)}$ is the black hole entropy in final (initial) state and $S$ is the object entropy, we obtain

$$
S \leq 4 \pi E_{T}-\frac{\pi e^{\prime 2}}{M}-\frac{2 \pi e^{\prime} q}{M} .
$$

When $M$ is large with respect to $e, q, m^{\prime}$, and $e^{\prime}$, we can expand the surface gravity

$$
\kappa=2 \sqrt{M^{2}-q^{2}} /\left(M+\sqrt{M^{2}-q^{2}}\right),
$$

as $\kappa \simeq 1-q^{2} /\left(4 M^{2}\right)+O\left(M^{-4}\right)$. In this limit the leading terms in the inequality (67) are independent of black hole's parameters

$$
S \leq 2 \pi m^{\prime} l+\frac{\pi e^{\prime 2}}{2} \frac{\mu^{2}}{\bar{\mu}^{2}} l^{2}+O\left(\frac{1}{M}\right) .
$$

This shows that the electrostatic self-energy modifies the Bekenstein upper bound.

In the massless Schwinger model, $\mu=\bar{\mu}$, the eq. becomes

$$
S \leq 2 \pi m^{\prime} l+\frac{\pi e^{\prime 2}}{2} l^{2}+O\left(\frac{1}{M}\right),
$$

which indicates the absence of the fermionic information in the upper entropy bound of the object. This agrees to the fact that near the horizon, massive gauge fields act like conformal massless fields. Besides the rôle of vacuum polarization, a main difference with respect to the result obtained in $Q E D_{4}$, 27, is the sign and the order of terms in right hand side of (69), in other words the bound is not tightened here. For $e^{\prime}=0$ we obtain the well known result 28]

$$
S \leq 2 \pi m^{\prime} l .
$$




\section{References}

[1] J. D. Bekenstein, Phys. Rev. D9, 3292 (1974).

[2] J. D. Bekenstein, Phys. Rev. D23, 287 (1981).

[3] J. D. Bekenstein and A. E. Mayo, Phys. Rev. D61, 24022 (2000).

[4] A. E. Mayo, Phys. Rev. D60, 104044 (1999).

[5] H. Mohseni Sadjadi, Gen. Rel. Grav. 37, 281 (2005); ibid. 36, 199 (2004).

[6] J. Schwinger, Phys. Rev. 128, 2425 (1962).

[7] S. Coleman, R. Jackiw and L. Susskind, Ann. Phys. 93, 267 (1975).

[8] R. Gass, Phys. Rev. D27, 2893 (1983).

[9] J. Barcelos-Neto and A. Das, Phys. Rev. D33, 2262 (1986).

[10] O. J. P. Eboli, Phys. Rev. D36, 2408 (1987).

[11] A. Ghosh, hep-th/9604056

[12] H. Mohseni Sadjadi and Kh. Saaidi, Phys. Rev. D63, 65009 (2001).

[13] M. Alimohammadi and H. Mohseni Sadjadi, Phys. Rev. D63, 105018 (2001).

[14] E. Abdalla, M. C. Abdalla, and K. D. Rothe, Non-perturbative methods in 2 dimensional quantum field theory (World Scientific, Singapore, 1991).

[15] D. J. Gross, I. R. Klebanov, A. V. Matystin, and A. V. Smilga, Nucl. Phys. B461, 109 (1996).

[16] V. Frolov, S. V. Sushkov, and A. Zelnikov, Phys. Rev. D67, 104003 (2003).

[17] A. Armoni, Y. Frishman, and J. Sonnenschein, Int. J. Mod. Phys. A14, 2475 (1999).

[18] A. G. Smith and C. M. Will, Phys. Rev. D22, 1276 (1980).

[19] B. Carter in General Relativity: An Einstein Centenary Survey, editors S. W. Hawking and W. Israel (Cambridge University Press, New York, 1979).

[20] http://functions.wolfram.com

[21] I. Sachs and A. Wipf, Phys. Lett. B326, 105 (1994).

[22] Nail R. Khusnutdinov, math-ph/0302054

[23] A. Erdélyi and H. Bateman, Higher transcendental functions, V.1 (McGraw-Hill Book Company Inc., New York, 1953).

[24] B. Linet, Phys. Rev. D61, 107502 (2000).

[25] V. P. Frolov, Phys. Rev. D46, 5383 (1992).

[26] J. Ho, Phys. Rev. D64, 64019 (2001).

[27] O. Zaslavskii, Gen. Rel. Grav. 24, 973 (1992).

[28] S. Mignemi, Phys. Rev. D70, 047501 (2004). 